\title{
Monte Carlo simulations of a single polymer chain under an external force in two and three dimensions
}

\author{
M. Wittkop, J.-U. Sommer, S. Kreitmeier, and D. Göritz \\ Institute of Applied Physics, University of Regensburg, D-93040 Regensburg, Germany
}

(Received 23 June 1993; revised manuscript received 17 January 1994)

\begin{abstract}
The deformation behavior of a single polymer chain subjected to an external force was studied by computer simulations. Both random walks and self-avoiding walks were investigated. The simulations were performed in two and three dimensions using the bond fluctuation model. The projection of the end-to-end vector in the force direction as a function of the applied force was compared to the scaling function obtained from renormalization group studies, covering the full interesting force regime. The differences in the crossover between the linear force and Pincus-scaling regime were studied.
\end{abstract}

PACS number(s): 64.70.-p, 05.40.+j, 83.10.Nn, 83.20.Jp

\section{INTRODUCTION}

The excluded volume or self-avoiding effect of polymer chains is one of the most discussed problems in polymer physics. The theoretical understanding has made great progress and the mathematical techniques are well developed. They can be read in many publications or surveys, cf. $[1-3,6]$.

If an external force is applied to a single polymer chain the deformation behavior of such a chain is quite different from a Gaussian one $[4,5]$. A scaling solution of this problem was first given by Pincus using the blob picture [7]. However, such a scaling discussion can only describe the limiting cases of weak and strong forces but not the behavior between both regimes. To get the behavior over the full force range it is necessary to use a more detailed mathematical analysis.

The starting point is the partition function of a continuous chain with both ends subjected to an external force in opposite directions:

$$
Z(\mathbf{f})=\bar{Z}_{0} \int \mathrm{d}^{d} R P(\mathbf{R}, L, l) \exp \{\mathbf{f} \cdot \mathbf{R}\} .
$$

$P(\mathbf{R}, L, l)$ is the exact Green's function of the excluded volume chain. $L$ is the "contour length" of the chain, $l$ the elementary step length, and $\mathbf{R}$ the vector connecting both chain ends. f $k_{B} T$ represents the applied force. $\bar{Z}_{0}$ stands for the number of configurations of the excluded volume chain without any further constraints and $d$ is the dimension of space.

Using Eq. (1) the mean value of the projection of the end-to-end vector in force direction $\left\langle R_{f}\right\rangle$ can be calculated as

$$
\left\langle R_{f}\right\rangle=-\frac{1}{k_{B} T} \frac{\partial F}{\partial f}=\frac{\partial \ln (Z(\mathbf{f}))}{\partial f} .
$$

$F$ is the free energy as a function of the force: $F=$ $-k_{B} T \ln (Z(\mathrm{f}))$. The Green's function of the excluded volume chain can be presented in the following scaling form, see for example Refs. [ 1 and 6$]$ :

$$
P(\mathbf{R}, L)=\frac{1}{X^{d}} h\left(\frac{R}{X}\right) .
$$

The Green's function of a free excluded volume chain is isotropic. Thus, the function $h$ is only a function of the absolute value of the end-to-end vector. $X$ is defined as

$$
\left\langle\mathbf{R}^{2}\right\rangle=N^{2 \nu} l^{2}=X^{2} d,
$$

where $\nu$ is the critical exponent and $N$ the number of segments. For large $x$ the function $h(x)$ can be approximated in the following manner [6]:

$$
h(x) \sim x^{\kappa} \exp \left(-D x^{\delta}\right),
$$

where $D$ is introduced for a proper normalization of the moments. The exponents $\delta$ and $\kappa$ scale according to the following scaling relations $[9,10]$ :

$$
\delta=\frac{1}{1-\nu}, \quad \kappa=\frac{1-\gamma+\nu d-d / 2}{1-\nu},
$$

where $\gamma$ is a critical exponent $[6,8]$.

We now assume that the external force has only a component in $z$ direction. Furthermore, we define $f=f X$. In three dimensions $(d=3)$, we get, after performing the angle integrations,

$$
Z(\mathbf{f}) \sim 4 \pi \int_{0}^{\infty} \mathrm{d} x x^{1+\kappa} \exp \left(-D x^{\delta}\right) \frac{1}{\tilde{f}} \sinh (x \tilde{f}) .
$$

If this integral is dominated by a sharp maximum, we can use a saddle point approximation. This approximation is $\operatorname{good}$ if $\tilde{f} \gg 1$, i.e.,

$$
f>X^{-1} \quad \text { or } \quad f>\frac{1}{N^{\nu l}} .
$$

The saddle point approximation yields the well-known result for the averaged deformation in direction of the forces: 


$$
\left\langle R_{f}\right\rangle \sim-\frac{\partial F}{\partial \tilde{f}} X \sim X \tilde{f}^{\frac{1}{\nu}-1} \sim N l(f l)^{\frac{1}{\nu}-1} .
$$

In the case of $f<1 /\left(N^{\nu} l\right)$ the saddle point approximation is no longer valid. A better way is to expand here the exp function of Eq. (1). The result is

$$
\left\langle R_{f}\right\rangle=\frac{1}{d}\left\langle\mathbf{R}^{2}\right\rangle f=\frac{1}{d} N^{2 \nu} l^{2} f .
$$

Note that Eq. (7) is only correct in three dimensions, but the scaling laws Eqs. (9) and (10) are also valid in two dimensions.

An experimental verification of the results, especially of Eq. (9), is, in contrast to the undeformed state, quite difficult. With some idealizations the deformation of ideal chains, as they are represented in polymer melts, can be observed in the deformation behavior of polymer networks. But the network chains cannot be separated from each other. Even totally swollen networks can only be regarded as semidilute. Real polymer networks exhibit additionally a large polydispersity of the network chain lengths [12]. Another possibility to study the deformation behavior are flow experiments of dilute polymer solutions in extreme shear gradients as already proposed by de Gennes [1]. But in that case hydrodynamic effects must be included. Therefore, the interpretation of such results would be quite difficult with regard to Pincus scaling.

In this situation computer experiments are almost the only reliable and simple possibility to check the theoretical results. Computer simulations have shown their great possibilities, for example, in investigating the unstretched state of single random walks and self-avoiding walks (Baumgärtner, Binder, and co-workers [13]) or in calculating some aspects on the deformation of short chains, i.e., the transportation of force through the backbone, excluded volume contacts, etc., and the force fluctuations in time (Weiner and co-workers [14]). Also, the mechanical properties of networks were studied extensively under various viewpoints (Gao, Weiner, and Termonia [15]). Polymer melts and glasses were simulated mainly by molecular dynamics [16]. At $T=0 \mathrm{~K}$ the mechanical properties of polypropylene were computed by energy minimizations (Suter and co-workers [17]). Molecular dynamics simulations on the deformation of glasses show similarities between these short time ( $\sim 1 \mathrm{ns)} \mathrm{sim-}$ ulations and laboratory measurements obtained on time scale orders of longer magnitude [18]. Dickman and Hong [19] simulated the force between grafted polymeric brushes.

Directly related to our topic is a work of Webman, Lebowitz, and Kalos [5]. They have observed the Pincus scaling in three dimensions. Also, the weak force regime has been obtained. The crossover region between both scaling regimes appears very narrow in contrast to the first order renormalization group calculations given by Oono et al. [4]. On the other hand, this first order calculation is not very convincing with respect to this topic, so that a decision about these facts as well as a quantitative analysis over the full force range are outstanding up to now.
A problem, which arises in the comparison of the simulated results and the theoretical results, is due to the fact that the continuous chain model used so far is infinitely stretchable. The scaling behavior obtained in Eq. (9) is no longer valid if the Pincus blobs [7] are of the order of the real statistical segments [11], i.e., $f \sim l^{-1}$. In this case the scaling law breaks down and the response of the chain is governed by the orientation entropy of rigid, free rotating, and independent statistical segments, which can be described by means of a Langevin function.

In this strong force region the microscopic properties begin to influence the behavior, therefore, it is interesting to know the exact solution for the bond fluctuation model. Generally for the case of a lattice model the polymer segment cannot rotate freely. Some corrections to the Langevin function appear if the exact partition function for the lattice model is calculated regardless of the monomer interactions.

Calculating the exact partition function in the bond fluctuation model with the use of Eq. (2) leads to

$$
\left\langle R_{f}\right\rangle=N \mathcal{L}_{\mathrm{BFM}}(f)
$$

with

$$
\mathcal{L}_{\mathrm{BFM}}(f)=\frac{\sum_{k=1}^{M_{B}} b_{f}^{(k)} \exp \left\{f b_{f}^{(k)}\right\}}{\sum_{k=1}^{M_{B}} \exp \left\{f b_{f}^{(k)}\right\}},
$$

where $b_{f}^{(k)}$ is the projection of the bond vector in the force direction. $k$ runs from 1 to $M_{B}$, with $M_{B}$ the number of bond vectors (for example, $M_{B}=108$ in three dimensions). The function $\mathcal{L}_{\mathrm{BFM}}(f)$ is a generalization of the classical Langevin function for the bond fluctuation model and is easy to compute numerically. The function $\mathcal{L}_{\mathrm{BFM}}(f)$ is appropriate to test the strong force properties of a chain with excluded volume and the behavior of random walks simulated by the bond fluctuation model.

\section{SIMULATION OF THE DEFORMATION BEHAVIOR OF A SINGLE CHAIN}

We used the bond fluctuation model in two and three dimensions. On the lattice the monomers are represented by plaquettes, respectively, cubes of $2^{d}$ places connected by a set of possible bond vectors ( 36 in two dimensions and 108 in three dimensions). The diffusion dynamics is simulated by randomly chosen jumps (accepted by checking certain conditions) of the monomers in the spatial directions. By forcing self avoiding of the monomers excluded volume is fulfilled (this leads automatically to cut avoiding for the used set of bonds). The simulation was athermal since no interactions between the monomers were taken into account. For more details of the bond fluctuation model we refer to the original papers [20].

We have studied chains of $(N+1)=20,40,60,80$, and 100 monomers on lattices with periodic boundary conditions. In two dimensions the lattice extensions were 


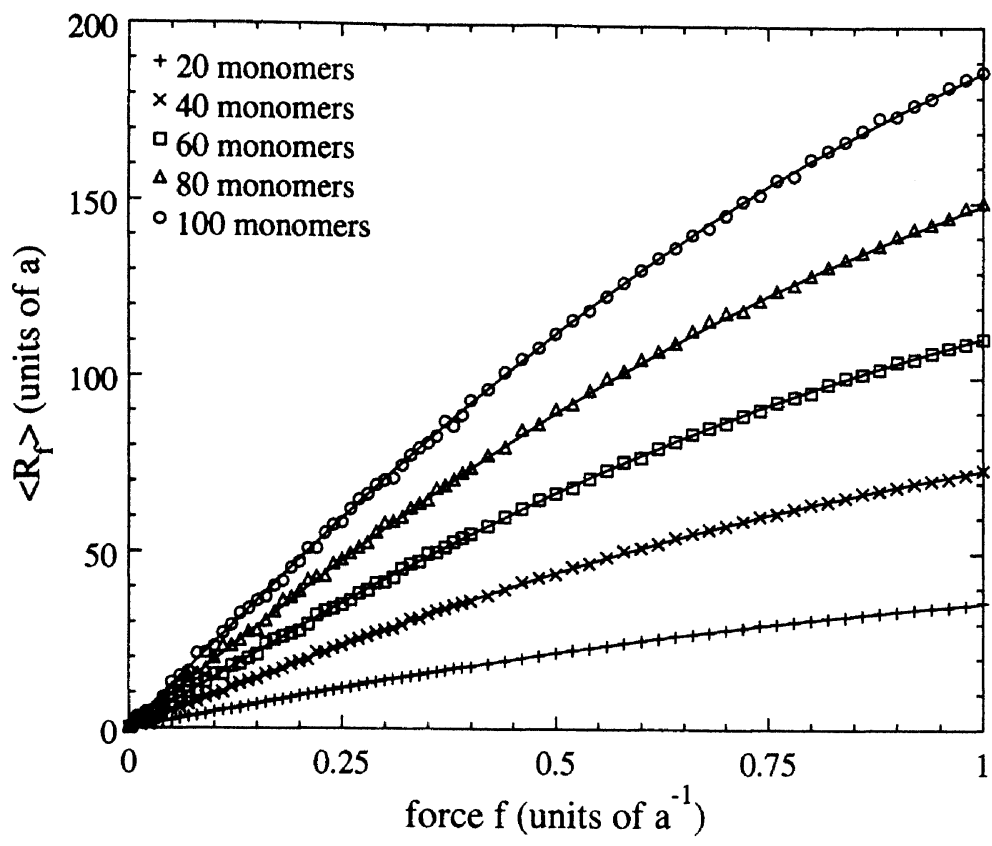

FIG. 1. Comparison of $\left\langle R_{f}\right\rangle$ measured during the simulations (points) and $N \mathcal{L}_{\mathrm{BFM}}(f)$ (solid lines). From bottom to top: $(N+1)=20,40,60,80,100$ monomers.

$40 \times 120$ up to $100 \times 300$. In three dimensions we used $20 \times 20 \times 50$ up to $48 \times 48 \times 176$ lattices. The force was applied in $y(d=2)$ respectively $z(d=3)$ direction. The lateral dimensions of the lattices are large enough so that perturbations of the chain with itself over the periodic boundary conditions can be neglected. To improve the statistics we have performed 100 independent simulations for each chain length.

After creating and relaxing the chains we applied a force $f k_{B} T$ to the chain ends using the Metropolis algorithm [21]. We increased the force $f$ stepwise by $0.0025 a^{-1}\left(f \leq 0.05 a^{-1}\right), 0.005 a^{-1}\left(0.05 a^{-1}<f \leq\right.$ $\left.0.1 a^{-1}\right), 0.01 a^{-1}\left(0.1 a^{-1}<f \leq 0.4 a^{-1}\right)$, and $0.02 a^{-1}$ $\left(f>0.4 a^{-1}\right)$, where $a$ is the lattice spacing. In $d=2$ we added a regime with degree $0.001 a^{-1}\left(f \leq 0.01 a^{-1}\right)$. After each step we allowed the conformation to relax for 100000 MCS (1 MCS, one Monte Carlo step, is one attempted jump per monomer). Then the end-to-end vector $R_{f}$ in the force direction was measured in intervals of $500 \mathrm{MCS}$ for a total amount of $150000 \mathrm{MCS}$. Thus, $\left\langle R_{f}\right\rangle$ is averaged over 30000 samples.

In a test of the MC algorithm the excluded volume constraints were suppressed and the simulation results were compared with Eq. (11) (see Fig. 1). The good agreement between the points and the exact function $N \mathcal{L}_{\mathrm{BFM}}(f)$ confirms the applicability of the method to study chains under external forces. We also tested the assumption that for large enough forces the function $\mathcal{L}_{\mathrm{BFM}}(f)$ describes the deformation of chains with excluded volume [self-avoiding walks (SAW's)]. We verified this for strong forces $\left(f>a^{-1}\right)$.

\section{RESULTS AND DISCUSSION}

In this section the results of the simulation of SAW's will be compared with the theoretical calculations. We use the numerically obtained function $\left\langle R_{f}\right\rangle(f)$ by integration of the general scaling function $P(\mathbf{R}, L, l)$ described by Eqs. (3) and (5). [Corrections to the Cloizeaux-(Fisher-McKenzie-Moore) scaling Eq. (5) can be taken into account if the $R$ is outside a certain interval $\left[R_{*}, R_{* *}\right][8] . R_{*}$ is given by the relation

$$
\bar{v}_{0}\left(R_{*}^{2} / l\right)^{1 / 2} \sim 1
$$

with $\bar{v}_{0}=v_{0} l^{-2}(d / 2 \pi l)^{d / 2}$, where $v_{0}$ is the excluded volume strength. $R_{*}$ turns out to be of the order of the lattice spacing and is, therefore, not of interest for the considered force regime. The quantity $R_{* *}$ is far beyond the "downturn" regime where the finitely extensibility influences the behavior and is, therefore, also not interesting.] It is quite complicated to calculate the value $D$ of Eq. (5) directly, but it is a single parameter which has to be the same for each chain length, so we introduce further reduced values $\bar{f}$ and $\bar{x}$ :

$$
\bar{x}=D^{\frac{1}{\delta}} x=D^{\frac{1}{\delta}} \frac{R}{X} \quad \text { and } \quad \bar{f}=D^{-\frac{1}{\delta}} \tilde{f}=D^{-\frac{1}{\delta}} X f .
$$

For $d=3$, we get in this way

$$
\left\langle\bar{x}_{f}\right\rangle=\frac{1}{\bar{f}}\left(\frac{\int_{0}^{\infty} \mathrm{d} \bar{x} \bar{x}^{2+\kappa} \exp \left(-\bar{x}^{\delta}\right) \cosh (\bar{x} \bar{f})}{\int_{0}^{\infty} \mathrm{d} \bar{x} \bar{x}^{1+\kappa} \exp \left(-\bar{x}^{\delta}\right) \frac{1}{\bar{f}} \sinh (\bar{x} \bar{f})}-1\right) .
$$

In the same manner we have for $d=2$,

$$
\left\langle\bar{x}_{f}\right\rangle=\frac{\int_{0}^{\infty} \mathrm{d} \bar{x} \bar{x}^{2+\kappa} \exp \left(-\bar{x}^{\delta}\right) \int_{0}^{2 \pi} \mathrm{d} \varphi \exp [\bar{x} \bar{f} \cos (\varphi)] \cos (\varphi)}{\int_{0}^{\infty} \mathrm{d} \bar{x} \bar{x}^{1+\kappa} \exp \left(-\bar{x}^{\delta}\right) \int_{0}^{2 \pi} \mathrm{d} \varphi \exp [\bar{x} \bar{f} \cos (\varphi)]} .
$$

The integrations in Eqs. (13) and (14) were done numerically applying NAG-routines (Mark 14) [22].

For the critical exponents $\nu, \delta$, and $\kappa$ that appear in Eqs. (13) and (14), we used the following values [6]:

$$
\nu=\frac{3}{4}, \kappa=0.625, \delta=4.0,
$$

in two dimensions and

$$
\begin{aligned}
& \nu=0.588 \pm 0.001, \kappa=0.249 \pm 0.011, \\
& \delta=2.427 \pm 0.006
\end{aligned}
$$

in three dimensions. In Figs. 2(a) $(d=2)$ and 2(b) $(d=3)\left\langle\bar{x}_{f}\right\rangle$ is plotted versus $\bar{f}$ in both the theoretical 
functions Eqs. (14) and (13) (solid line) and the simulations (points). The only free parameter is $D$. We have fitted it so that the theoretical values match the experimental ones. $D$ does not change the behavior of the curves in the log-log plot in Figs. 2(a) and 2(b), but only shifts the values. With $D=0.11$ for $d=2$ and $D=0.35$ for $d=3$ we got the best agreement. Note that lattice artifacts are also included in $D$. Thus, the presented values of $D$ may differ from values of off-lattice calculations. At low forces the statistical fluctuations become larger. Nevertheless, it can be clearly seen that the shape of the theoretical curve - for instance, in the cross over regime - is in good agreement to the simulated values. At strong forces the simulated values are below the theoretical curve and the scaling breaks down since the chains
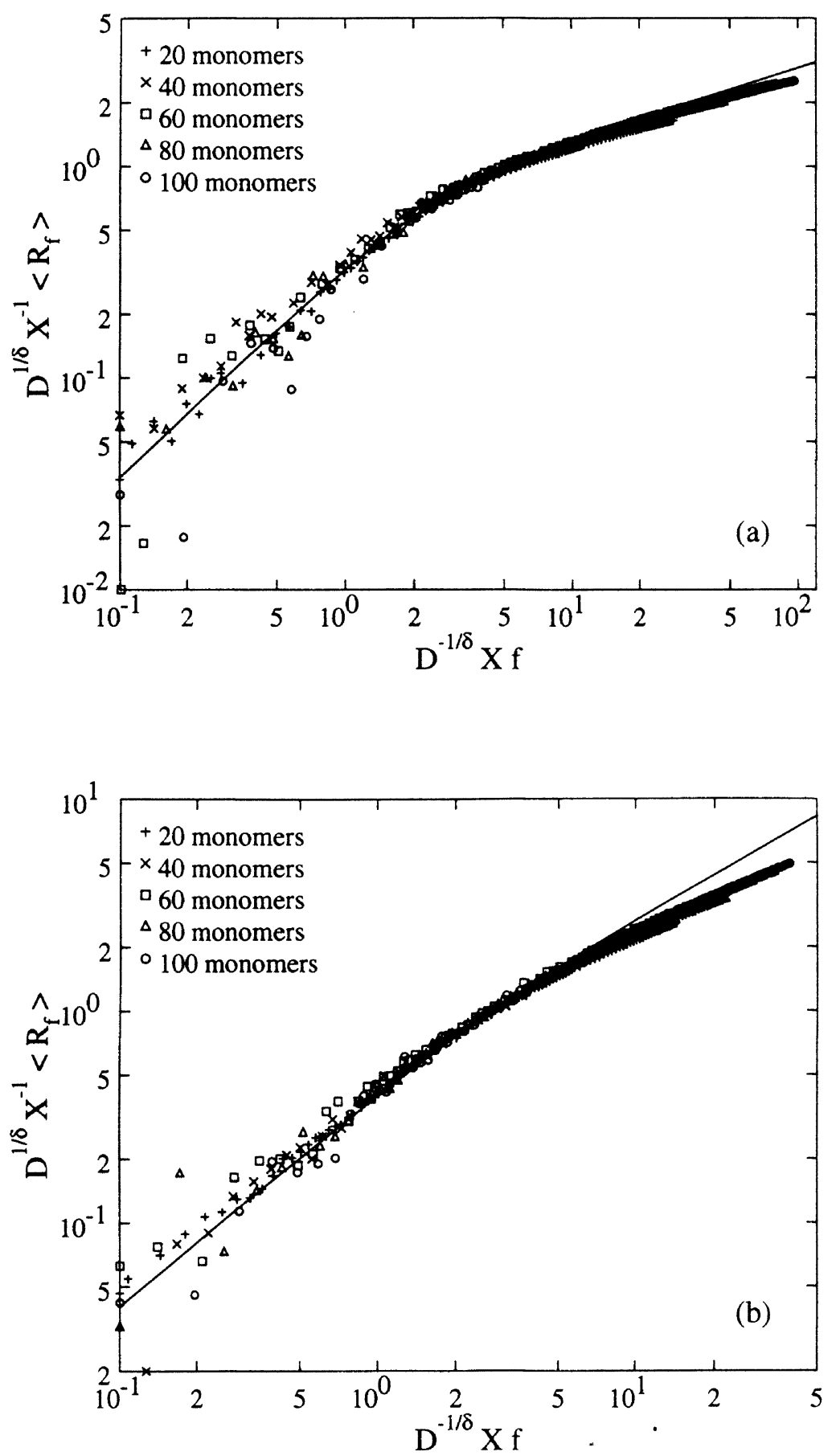

FIG. 2. (a) and (b) $\left\langle\bar{x}_{f}\right\rangle=D^{\frac{1}{\delta}} \frac{\left\langle R_{f}\right\rangle}{X}$ versus $\bar{f}=D^{-\frac{1}{\delta}} X f$ both the theoretical function (solid line) and the simulations (points) for various chain lengths in two (a) and three (b) dimensions. The parameters are $\kappa=0.625, \delta=4.0, D=0.11$ for $d=2$ and $\kappa=0.249, \delta=2.427, D=0.35$ for $d=3$. are not infinitely stretchable. The Pincus-scaling Eq. (9) should describe the deformation behavior in an intermediate force region $N^{-\nu} l^{-1}<f<l^{-1}$. In the bond fluctuation model - where $l$ is the mean bond vector length $-l^{-1}$ is $0.34 a^{-1}(d=2)$ and $0.37 a^{-1}(d=3)$. A lower limit for Eq. (9) is given by the condition $f>1 /\left(N^{\nu} l\right)$. In Figs. 3(a) $(d=2)$ and 3(b) $(d=3)\left\langle R_{f}\right\rangle$ is plotted versus $f$ for various chain lengths.

In both figures the simulated $\left\langle R_{f}\right\rangle$ (points) can be fitted by a single proportional factor $k$ in the scaling law Eq. (9) (solid lines). We used $k=0.635(d=2)$ and $k=0.455(d=3)$. Due to the fact that the lower limit of the range of validity is proportional to $1 / N^{\nu}$ the short chains reach this scaling law at higher forces. In contrast to that the upper limit $f \sim l^{-1}$ is independent of the
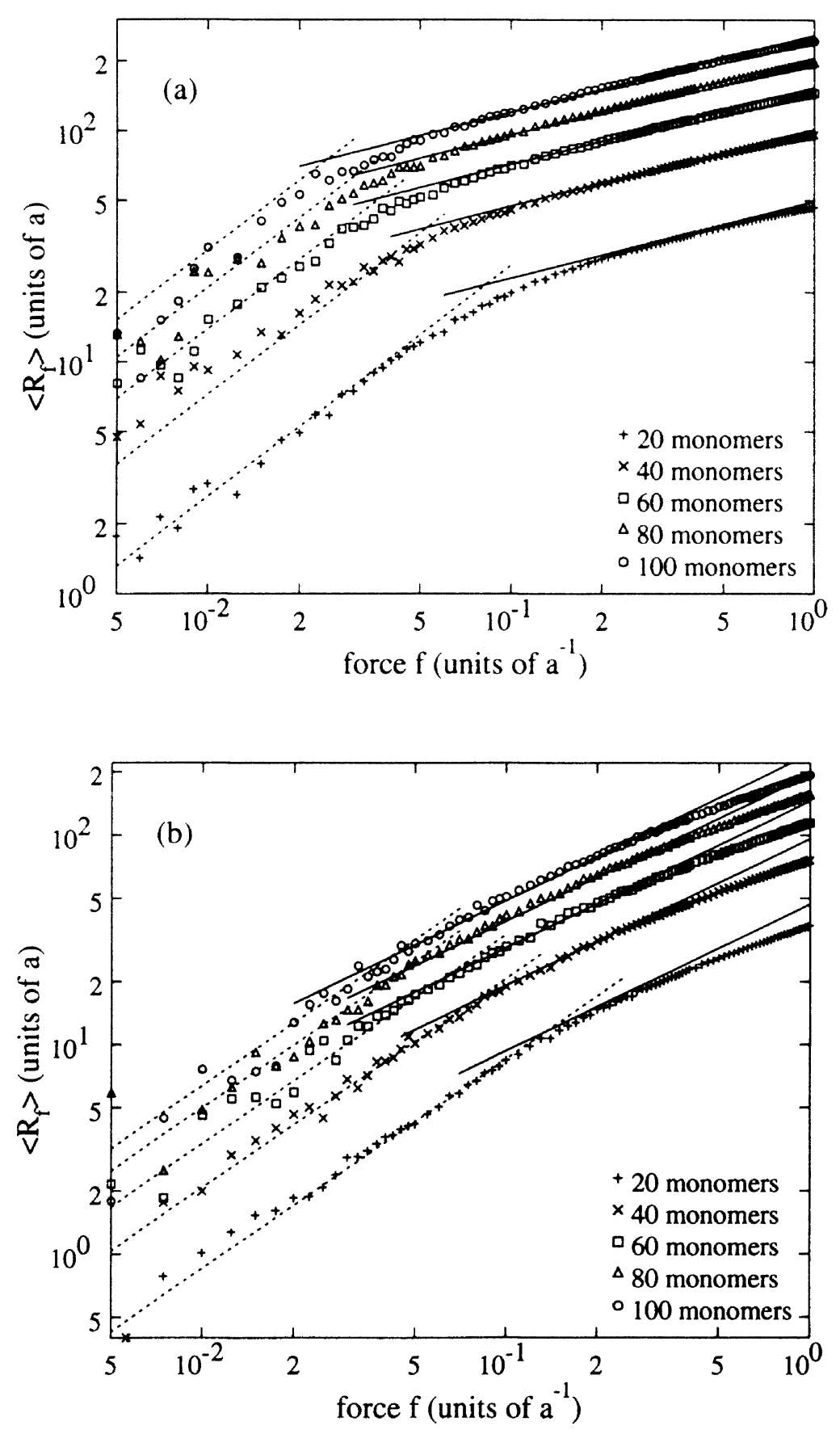

FIG. 3. (a) and (b) Comparison of the projected end-to-end vector $\left\langle R_{f}\right\rangle$ of the simulated SAW's (points) with the linear response $\left\langle R_{f}\right\rangle=\frac{1}{d}\left\langle\mathbf{R}^{2}\right\rangle f$ (dashed lines) and the Pincus-scaling law $\left\langle R_{f}\right\rangle=k N l(f l)^{\frac{1}{\nu}-1}$ (solid lines) in two (a) and three (b) dimensions. $k=0.635$ for $d=2$ and $k=0.455$ for $d=3$. 
numbers of monomers.

Also, the linear force behavior Eq. (10) is drawn in these figures (dashed lines). There is no fit parameter $\left(\left\langle\mathbf{R}^{2}\right\rangle\right.$ of the different chain lengths were computed in a separate simulation). Although the statistical fluctuations are large for small forces there is agreement between Eq. (10) and the simulated points.

The width of the crossover regime between the linear and the Pincus behavior differs between two and three dimensions (Figs. 2 and 3 ). In the case of two dimensions it is rather broad, whereas in three dimensions it becomes narrower. From the theoretical point of view the use of the scaling function in connection with the more exact critical exponents enforces a more abrupt crossover, compared to the first-order calculation of Oono et al. [4]

\section{ACKNOWLEDGMENTS}

Parts of the simulations were performed on the facilities of the Computer Center of the University of Regensburg. We thank them for the grant of computer time. J.U.S. thanks DFG for financial support (So 277/1-1).
[1] P.-G. de Gennes, Scaling Concepts in Polymer Physics (Cornell University Press, Ithaca, 1979).

[2] M. Doi and S.F. Edwards, The Theory of Polymer Dynamics (Clarendon Press, Oxford, 1988).

[3] Y. Oono and K. Freed, J. Chem. Phys. 75, 993 (1981); Y. Oono, T. Ohta, and K. Freed, J. Chem. Phys. 74, 6458 (1981).

[4] Y. Oono, T. Ohta, and K. Freed, Macromolecules 14, 880 (1981).

[5] I. Webman, J.L. Lebowitz, and M.H. Kalos, Phys. Rev. A 23, 316 (1981).

[6] J. des Cloizeaux and G. Jannink, Polymers in Solution (Oxford University Press, Oxford, 1989).

[7] P. Pincus, Macromolecules 9, 386 (1976).

[8] S. Stepanov, J. Phys. France 51, 899 (1990).

[9] M.E. Fisher, J. Chem. Phys. 44, 616 (1966).

[10] D.S. McKenzie and M.A. Moore, J. Phys. A 4, L-82 (1971).

[11] The symbol $l$ has, in the case of the continuous chain model, a quite formal meaning. It is reminiscent of the diffusion constant $D$ in continuous space diffusion.

[12] J.-U. Sommer, J. Chem. Phys. 95, 1316 (1991); M. Schulz and J.-U. Sommer, ibid. 96, 7102 (1992).

[13] A. Baumgärtner and K. Binder, J. Chem. Phys. 71(6), 2541 (1979); A. Baumgärtner, ibid. 72(2), 871 (1980); 73(5), 2489 (1980); Z. Phys. B. 42(3), 265 (1981); K. Kremer, A. Baumgärtner, and K. Binder, J. Phys. A 15(9), 2879 (1982); D. Ceperley, M.H. Kalos, and J.L. Lebowitz, Macromolecules 14, 1472 (1981); J. Baschnagel, K. Qin, W. Paul, and K. Binder, ibid. 25, 3117 (1992).

[14] J.H. Weiner and M.R. Pear, Macromolecules 10, 317 (1977); J.H. Weiner, ibid. 15, 542 (1982); J.H. Weiner and D.H. Berman, ibid. 17, 2015 (1984); J. Chem. Phys. 82(1), 548 (1985); J. Polym. Sci., Part B 24(2), 389 (1986).

[15] J. Gao and J.H. Weiner, Macromolecules 20, 2520 (1987); 20, 2525 (1987); 21, 773 (1988); 22, 979 (1989); 24, 1519 (1991); 24, 5179 (1991); Y. Termonia, ibid. 22, 3633 (1989); 23, 1481 (1990); 23, 1976 (1990); 24, 1392 (1991).

[16] A. Baumgärtner and K. Binder, J. Chem. Phys. 75(6), 2994 (1981); M. Bishop, D. Ceperley, H.L. Frisch, and M.H. Kalos, ibid. 72(5), 3228 (1980); K. Kremer and G.S. Grest, ibid. 92(8), 5057 (1990); D. Rigby and R.-J. Roe, ibid. 87(12), 7285 (1987); Macromolecules 22, 2259 (1989).

[17] D.N. Theodorou and U.W. Suter, Macromolecules 19, 139 (1986); 19, 379 (1986); P.H. Mott, A.S. Argon, and U.W. Suter, Polym. Prepr. 30(2), 34 (1989).

[18] D. Brown and H.R. Clarke, Macromolecules 24, 2075 (1991); J.I. McKechnie, D. Brown, and H.R. Clarke, ibid. 25, 1562 (1992); 26, 198 (1993); R. Cook, J. Polym. Sci., Part B 26, 1337 (1988); 26, 1349 (1988).

[19] R. Dickman and Daniel C. Hong, J. Chem. Phys. 95(6), 4650 (1991).

[20] I. Carmesin and K. Kremer, Macromolecules 21, 2819 (1988); A.L. Rodriguez, H.-P. Wittmann, and K. Binder ibid. 23, 4327 (1990); H.P. Deutsch and K. Binder, J. Chem. Phys. 94(3), 2294 (1991); H.-P. Wittmann, K. Kremer, and K. Binder, ibid. 96(8), 6291 (1992).

[21] N. Metropolis, A.W. Rosenbluth, M.N. Rosenbluth, A.H. Teller, and E. Teller, J. Chem. Phys. 21, 1087 (1953).

[22] The NAG Fortran Library Manual, Mark 14. Numerical Algorithms Group Limited, Oxford (1990). 